\title{
Architects and refugee camps: a case study for bottom-up approaches
}

\section{SIGRADI2018 TECHNOPOLITICAS \\ xxii congresso da sociedade iberoamericana de gráfica digital 22th conference of the iberoamerican society of digital graphics 07|08|09|novembro|2018 iau usp | são carlos | sp br}

\author{
Diogo Ribeiro Carvalho \\ PUC Minas | Brazil | diogocarvalho@pucminas.br
}

Taynara Vieira Deiró

PUC Minas | Brazil | Taynara_Deiro@hotmail.com

\begin{abstract}
The United Nations High Commissioner for Refugees (UNHCR) defines "refugees" as people forced to leave their country because of armed conflict, widespread violence and massive human rights violations. This paper presents and discusses a methodology for implementing a refugee camp in the context of the migration of southern Sudanese to Uganda. The proposal involves an intersection between 3D printing technology of LDM (Liquid Deposition Modeling) process, using soil as the main input, and participatory bottom-up processes in order to promote gradual technical and creative autonomy, sense of community and mental health of these people.
\end{abstract}

Keywords: Architecture; Refugee camps; Humanitarian design; Digital fabrication; Bottom-up approaches.

\section{INTRODUÇÃO}

Segundo dados do Alto Comissariado das Nações Unidas para os Refugiados (UNHCR, 2014), há atualmente cerca de 66,5 milhões de pessoas forçadas a sair de seu lugar de origem. Dentre esses, 22,5 milhões são refugiados, dos quais mais da metade possui menos de 18 anos. Trata-se de uma situação global alarmante e que exige cada vez mais ajuda humanitária, cujos pressupostos básicos são a preservação e a valorização da vida humana. Nesse cenário, organizações, instituições, empresas e pessoas físicas de variados campos disciplinares têm se mobilizado e atuado de modos distintos em auxílio às pessoas aqui de interesse. O UNHCR (2016) define "refugiados" como pessoas obrigadas a deixar seu país devido a conflitos armados, violência generalizada e violação massiva dos direitos humanos.

O Serviço de Inovação do UNHCR investiga, propõe e executa práticas de ajuda humanitária em que a inovação é construída através de abordagens bottom-up, fundadas em processos participativos e colaborativos, e de design centrado nas pessoas (Oslo, 2005). O estudo exploratório deste artigo é uma proposta de campo de refugiados em Uganda, realizado no contexto de um trabalho de conclusão de curso de Arquitetura e Urbanismo em uma instituição de ensino superior e que se desdobrou em pesquisa acadêmica. Frente às milhares de pessoas que atualmente se encontram na situação de refugiados, fazemos as perguntas: como arquitetura e design podem contribuir na construção de práticas de alteridade que favoreçam a manutenção dos direitos e das liberdades dos refugiados, tais como seus direitos à vida e à integridade pessoal? Como as disciplinas de design e criação facilitam e promovem a inovação - em termos de processos, produtos e/ou modos de relação - junto aos refugiados? Em que medida as novas tecnologias informacionais, de design e fabricação digital, informam e/ou potencializam a construção de autonomia técnicocriativa dos refugiados?

Algumas das principais publicações nesses campos disciplinares são: Expanding Architecture: Design as Activism (Bell, Wakeford, 2008); Beyond Shelter: Architecture and Human Dignity (Aquilino, 2011); Ground Rules in Humanitarian Design (AD Reader) (Chun, Brisson, 2015); Humanitarian Architecture: 15 stories of architects working after disaster (Charlesworth, 2015); Outlaw Territories: Environments of Insecurity/Architectures of Counterinsurgency (Scott, 2016); Displacements: Architecture and Refugee (Herscher, 2017); Making Heimat: Germany, Arrival Country: Atlas of Refugee Housing (Schmal, Elser, Scheuermann, 2017); Design for Good: A New Era of Architecture for Everyone (Cary, 2017). Observa-se que boa parte das publicações é muito recente, o que indica a novidade do tema no campo e a necessidade de expansão da discussão.

Atualmente, artistas como Tiffany Chung e Henk Wildschut; escritórios de arquitetura e design como Shigeru Ban, Estudio Teddy Cruz e No Mad Makers; organizações não governamentais como Architecture for Humanity e Pilosio Building Peace; grupos de pesquisa/extensão em instituições de ensino como o Massachusetts Institute of Technology, Oxford University e University College London; e ainda plataformas digitais como http://architectureforrefugees.com/ têm se comprometido com as causas dos refugiados a partir dos campos disciplinares da arte, da arquitetura e do design. Tais práticas são muito distintas e variam de acordo com o contexto de atuação, as metodologias, intenções e ideologias. Contudo, a grande maioria das soluções que têm sido utilizadas em abrigos emergenciais e em campos de assentamento são genéricas e 
implementadas de modo top-down (UNHCR, 2016). A partir dessa gama de abordagens foram realizadas análises críticas de seus modos e resultados para balizar estratégias futuras de avaliação, planejamento e execução de ações frente a questões de refugiados, e mesmo de outras comunidades em situações de risco. É pelo questionamento dos modos tradicionais que lidam com os refugiados que o estudo exploratório aqui discutido se fundamenta.

Assim, a proposta desse artigo é apresentar e discutir uma metodologia de abordagem de implementação da estrutura de um campo de refugiados no contexto da migração de sudaneses do sul para Uganda por conta do grave estado de guerra civil étnica do país. A proposta envolve interseção entre tecnologia de impressão 3D de processo LDM (liquid deposition modeling), utilizando terra como insumo principal, e processos participativos de caráter bottom-up na configuração espacial do campo de refugiados com vistas à promoção de autonomia técnicocriativa gradativa dessas pessoas, senso de comunidade e saúde mental.

Com uma durabilidade média de 17 anos, tais aglomerações começam a atingir escala de vilas e cidades. Constata-se em campos ao redor do mundo o surgimento de estruturas permanentes além de equipamentos de serviços e lazer que atestam a durabilidade e o surgimento de vínculos sociais dentro desses assentamentos. O alemão Kilian Kleinschmidt (In Radford, 2015), uma das maiores autoridades em ajuda humanitária, indica a urgência de se reconhecer o caráter permanente dos campos, uma vez que o tratamento desses espaços como temporários apenas contribui para a continuidade da situação de pobreza e limita as estratégias de suporte.

\section{METODOLOGIA}

A escolha da cidade de Arua como base para a proposta de implantação do modelo replicável de campo para um assentamento dos refugiados do Sudão do Sul se deu (1) pela cidade estar próxima à fronteira, mas a uma distância segura, segundo critérios do UNHCR (UNHCR, 2014); (2) por exibir opções de espaço livre próximo às áreas urbanas, permitindo a integração entre as populações; (3) por sediar quatro importantes instituições de ensino superior - Arua Technical Institute - Ragem (ATIR) (público), Uganda Christian University (privada), Islamic University in Uganda (comunitária), Muni University (público) - que podem potencializar o sucesso do campo em termos sociais, políticos e tecnológicos, além de já ser um destino comum aos refugiados sudaneses do sul.

As hipóteses construídas a partir de um mapeamento das abordagens dos campos da arquitetura e do design em relação aos refugiados e a partir da análise dos manuais do UNHCR são:

(1) Cada contexto exige soluções idiossincráticas - e mesmo em um mesmo contexto, situações diversas solicitam modos específicos de abordagem. A natureza essencialmente contextual dos campos de refugiados exige soluções idiossincráticas que trabalhem com a cultura das comunidades envolvidas, lógica que recentemente tem sido objeto de discussão no setor de
Serviço de Inovação do UNHCR e desafia as práticas humanitárias dos últimos 70 anos (UNHCR, 2017a; UNHCR, 2017b).

(2) A natureza permanente que os campos têm assumido exige uma abordagem distinta das tradicionais estratégias top-down que têm sido utilizadas para lidar com essas situações emergenciais. As abordagens mais eficazes são aquelas em que o refugiado é incorporado no processo de resolução de seus problemas (Collier, Betts, 2017) - incluindo a seleção de que problemas resolver isto é, as abordagens participativas e colaborativas de princípio bottom-up podem possibilitar uma maior autonomia da pessoa e, no âmbito da comunidade, a constituição de tecnologias sociais e a promoção de sustentabilidade nos campos de refugiados e na vida dos refugiados urbanos.

(3) Modos de sobreposição entre alta tecnologia e cultura local podem favorecer formas de diferenciação e expressão da identidade étnica e individual, e ainda facilitar a absorção de conhecimentos em tecnologias híbridas para futura aplicação. As impressoras LDM (liquid deposition modeling) permitem a customização das edificações por cada habitante, reforçando a sua autonomia sobre o processo de construção.

(4) O ensino de design thinking pode capacitar os refugiados para a concepção de modos de pensamento crítico-criativos e se mostrar como uma prática muito mais potente do que soluções prontas de terceiros, por princípio, top-down (Fairs, 2016). Esse tipo de acesso à educação (Spivak, 2012) é o grande diferencial que possibilita a transformação da condição de refugiado em não refugiado.

Assim, o estudo de caso e a pesquisa que daí se desdobra se justificam pelo potencial de amplificação da discussão no meio acadêmico em primeiro momento e sua repercussão reflexiva nas micropolíticas de gestão das/nas comunidades de refugiados.

\section{RESULTADOS}

\section{O LUGAR E O PROJETO}

Localizado em um terreno a 50 quilômetros da fronteira com o Sudão do Sul, na cidade de Arua em Uganda, com cerca de 620 mil metros quadrados, o projeto de reassentamento foi idealizado com estruturas e estratégias que permitam a integração dos refugiados sulsudaneses com a comunidade receptora. Muitas das diretrizes seguidas para dar forma à proposta estão presentes nos manuais fornecidos pelo UNHCR para orientar a construção e a manutenção dos campos de refugiados ao redor do mundo. A conexão com a comunidade local, a limitação da população ao número de vinte mil pessoas e a proposição de abrigos de baixo custo e impacto foram alguns norteadores extraídos dos guias técnicos (UNHCR, 2017c; UNHCR, 2017d). 


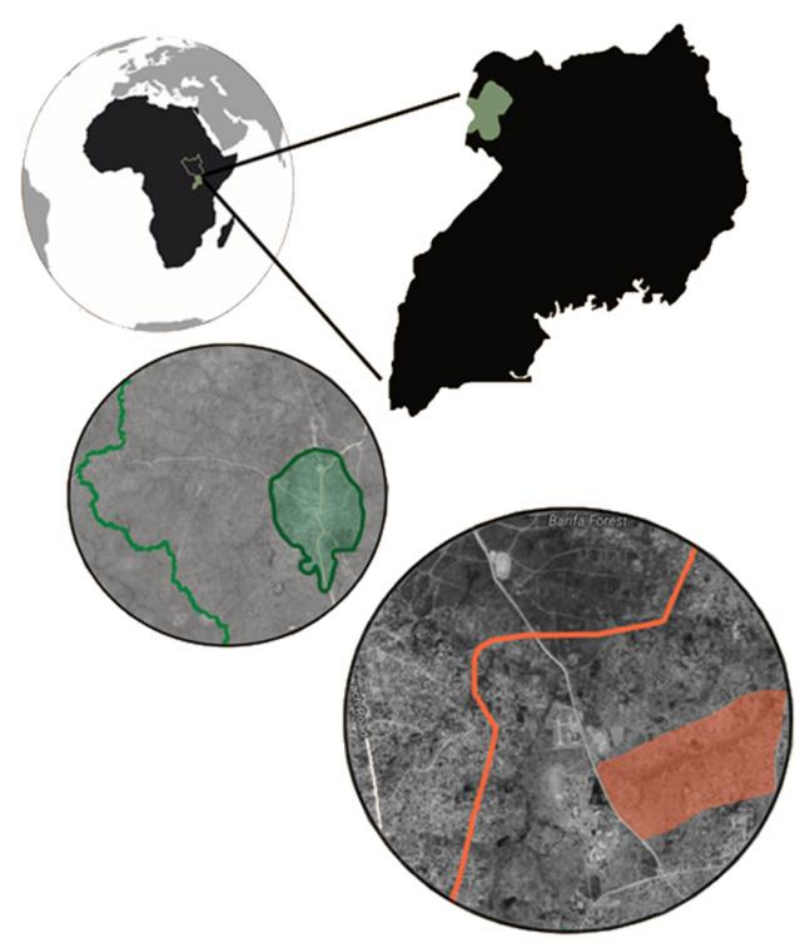

Figura 1: Delimitação geográfica do contexto, em Arua, Uganda. Fonte: Autores.

A lógica organizacional do assentamento se fundamenta em micro comunidades de quatro famílias, onde cada grupo tem direito a um lote com espaço para cultivo de alimentos e criação de animais, e uma habitação. No encontro dos quatro espaços unifamiliares há uma área comum destinada ao uso exclusivo das famílias circundantes. Esta área serve para promover a interação entre os vizinhos, além de ser o local destinado ao plantio de uma semente de árvore da flora local que cada micro comunidade recebe ao ser formada. $\mathrm{O}$ ato do plantio da árvore e a necessidade de cuidado para seu desenvolvimento - pré-requisito para a permanência da família ou grupo no campo - simbolicamente forjam um acordo informal de reciprocidade que potencializa a ajuda mútua e sensações de estabilidade e segurança (Scott, 2016). Como a árvore eventualmente florescerá por sobre as edificações, e o pátio interno do conjunto de quatro habitações pode ser visto tanto por áreas públicas, intermediárias e privadas, esses elementos servirão como um termômetro da qualidade das relações nessa micro comunidade.

\section{ESTRATÉGIAS}

A opção de se trabalhar primeiro na pequena escala se baseia na premissa de que se as micro relações são bem desenvolvidas e consolidadas, elas podem se autorreplicar de modo transescalar, potencializando um crescimento saudável (Heroux, Norris, Rube, Nadimi, 2017), mesmo em meio à situação de crise. Criar espaços de socialização em que os envolvidos possam intervir diretamente na sua criação e manutenção, não é uma forma de se ocupar a mente e de propor interação entre as pessoas, mas é uma estratégia de construção de autonomia. Pieter Ventevogel (2017), chefe de saúde mental do UNHCR, aponta que as intervenções mentais mais poderosas se relacionam ao empoderamento de pessoas e ao fortalecimento das comunidades de refugiados, pois estas são artificiais por se formarem ao acaso. Por isso Ventevogel defende estratégias de recriação de conexões sociais como primeira medida a se tomar em um campo.

Empoderar significa criar meios para que as pessoas sejam autônomas, e é determinante na abordagem bottom-up proposta para a implementação desse campo em Arua. Em divergência com os procedimentos padrões de construção de campos de refugiados ou de assentamentos pós-catástrofe, a proposta busca colocar o refugiado como agente ativo na produção das habitações e equipamentos coletivos pela implantação de canteiros de obra que funcionam como laboratórios de fabricação digital. Kilian Kleinschmidt (In Radford, 2015) aponta que as organizações humanitárias precisam entender o potencial que as novas tecnologias como a impressão 3D podem ter na construção da autonomia das pessoas em questão. No estudo exploratório deste artigo, esse processo ocorre por meio da capacitação dos refugiados para montar e operar impressoras $3 \mathrm{D}$ de grande porte para a confecção de suas habitações. Muito além da aprendizagem de um novo maquinário, cada vez mais relevante no universo da construção e do design, colocar os habitantes no topo da produção permite maior engajamento dos mesmos com o espaço físico em que estão sendo inseridos, de forma compulsória, um maior envolvimento com a nova comunidade e um maior senso de pertencimento. Se um refugiado aprende o processo e a técnica, ele não só pode ensinar outros tantos, como pode avançar para a etapa de design das estruturas.

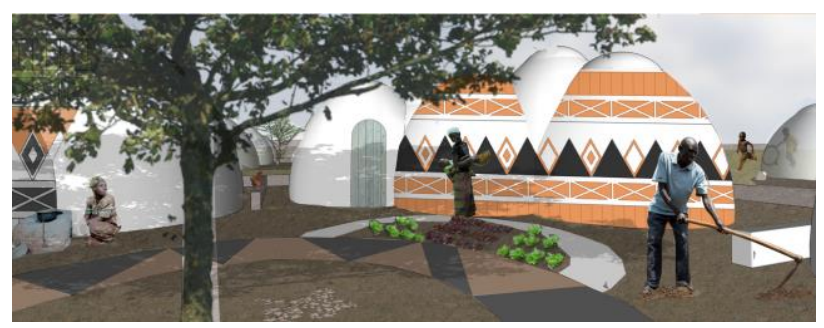

Figura 2: Pátio Interno comum à micro comunidade. Fonte: Autores.

\section{TECNOLOGIA CONSTRUTIVA}

Impressoras como a BigDelta da companhia WASP World's Advanced Saving Project - são os suportes tecnológicos chaves para a execução da proposta de forma eficiente, especialmente pelo seu fundamento Open Source (WASP, n/d). A necessidade de se construir o maior número de residências no menor tempo possível é um parâmetro que o UNHCR considera importante, assim como a preferência pela possibilidade de autoconstrução da habitação. No entanto, no caso em questão, a necessidade de produção em larga escala não se sobrepôs à urgência de se propor estruturas mais humanas, menos genéricas. Pelo contrário, a utilização de uma tecnologia construtiva que permite a variabilidade no design, sem comprometer custo e tempo, possibilitou que a proposta aliasse as duas demandas, indiscutíveis para a viabilidade do projeto.

Esse modelo de impressora 3D se mostra viável pois utiliza materiais de custo baixo, como terra/barro e palha, e ainda constrói em um tempo recorde em relação a construções feitas apenas com a mão de obra humana, com alta precisão, poupando recursos que podem ser utilizados em outra necessidade humanitária. Esta 
precisão também permite a proposição de arquiteturas com formas variadas, superfícies com cavidades ou protuberâncias que podem gerar padrões simples ou complexos, que demandam muito tempo de execução se feitos de modo artesanal (Keep, n/d).

A escolha da terra molhada misturada a palha como material base das edificações do reassentamento visa uma série de benefícios para seus habitantes. As paredes grossas de estruturas de barro, de alta inércia térmica, proporcionam qualidades termo acústicas que trazem conforto e privacidade. O barro é um elemento construtivo extremamente barato, e por esta razão a arquitetura de terra faz parte da cultura construtiva tradicional de diversos povos africanos, inclusive no Sudão do Sul. O material reforça o caráter ambientalmente sustentável da proposta na medida em que terá baixo impacto nas condições naturais do terreno escolhido, pois a construção das três mil habitações, previstas na área de 620 mil metros quadrados, demanda a escavação de apenas $2 \mathrm{~cm}$ em relação à superfície total.

No sistema construtivo de processo LDM (liquid deposition modeling) o insumo pastoso é depositado em camadas horizontais que se sobrepõem ao longo do tempo, e exige que a estratégia estrutural, em especial para se cobrir o espaço, se fundamente na lógica dos arcos, das abóbadas e cúpulas, que atuam bem sob compressão. Formas arquitetônicas com esses elementos reiteram as características culturais construtivas tradicionais da população de refugiados no reassentamento. Assim, a escolha de materiais e sistemas já conhecidos permite a criação de um vínculo mais rápido com a moradia e o "novo" modo de morar (Herscher, 2017), além de deixar os moradores mais confortáveis para construírem anexos e fazerem pequenas reformas, para que estas se adequem cada vez mais às realidades individuais.
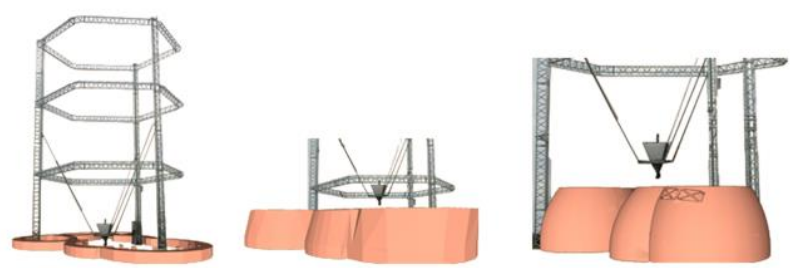

Figura 3: Processo construtivo LDM com uma estrutura delta. Fonte: Autores.

A política de gestão do campo de refugiados proposto para Arua inclui a possibilidade de escolha pela família ou grupo refugiado do design da habitação, em escala compatível à quantidade de pessoas, e do padrão ornamental das superfícies. O ornamento é tomado como estratégico para a proposta por se mostrar um eficiente portador de histórias, ideias, mitos, fatos e representações da vida (Carvalho, 2016). O ornamento é historicamente considerado um receptáculo de significados, isto é, tem um potencial simbólico enorme, cuja recepção não é unívoca nem necessariamente imutável, mas possui uma condição semântica metamórfica que varia com o tempo, a experiência e a cultura (Bloomer, 2000). É considerando o caráter ontológico intrínseco ao ornamento, que ele é utilizado como estratégia de potencialização do sentimento de pertencimento, diluindo as sensações de vazio, medo e desenraizamento que a situação de refugiado traz (Bauman, 2017).

No intuito de explorar ao máximo as potencialidades da técnica construtiva, a infraestrutura básica de mobiliário, tais como camas, bancadas, fogão e bancos, é pensada para ser embutida na estrutura e executada com impressão 3D. Esta estratégia elimina a necessidade de investimentos extras em infraestrutura e dispensa técnicas construtivas adicionais.

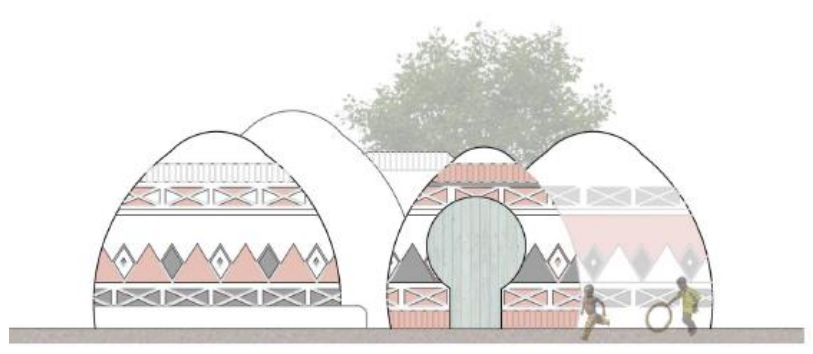

Figura 4: Vista da habitação e suas superfícies ornamentais. Fonte: Autores.

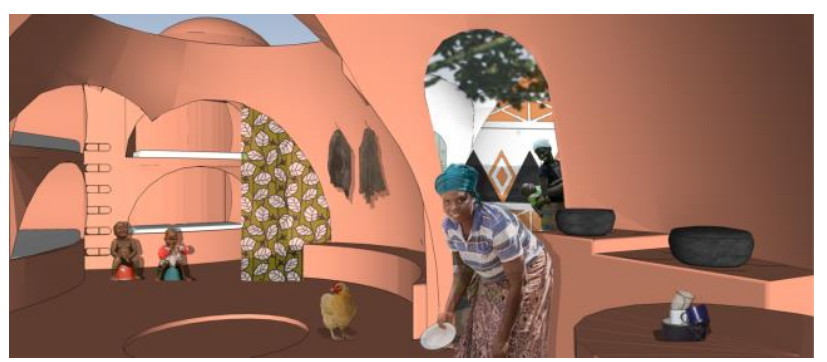

Figura 5: Vista interna da habitação. Fonte: Autores.

\section{DISCUSSÃO}

Todas essas condições, a estratégia tecnológica, a estética ornamental, a liberdade de escolha formal da habitação, a formação de micro comunidades e a instauração de vínculo simbólico com o lugar, contribuem para o movimento conceitual do termo abrigo para habitação e, em pouco tempo, para casa/lar. Assim, as estruturas arquitetônicas serão designadas como casas, em detrimento do termo 'abrigo', usualmente utilizado no campo do design humanitário. A proposta para Arua apresenta uma possibilidade de habitação que concede muito mais do que apenas espaço de sobrevivência. Ela oferece infraestrutura suficiente para proporcionar independência aos seus moradores. À medida que tais estruturas forem autoconstruídas, ocupadas e alteradas, estarão deixando de ser habitáculos para se converterem em casas e lares: passarão a carregar um pouco da identidade dos seus habitantes. A proposta tecnológica, aliada à política de gestão bottom-up se mostra como uma potente prática de alteridade (Boff, 2008; Capra, Luisi, 2014).

O caráter não efêmero das habitações, estratégia ainda bastante criticada nas políticas tradicionais de refugiados e nos governos que os recebem, apesar do tempo médio de existência de um campo ser de 17 anos, se justifica na verdade como o principal atrativo para os estados disponibilizarem espaço para 0 assentamento. A possibilidade de reaproveitamento das estruturas como 
habitação social, com o fim do campo, gera retorno social e financeiro para a comunidade receptora. Caso não haja necessidade de se manter as casas, basta a sua demolição sem causar déficit financeiro e impacto ambiental: a terra volta à terra, o material orgânico retorna à sua condição originária.

A impressão 3D de casas já é realidade (Gramazio, Kohler, 2014), contudo, a utilização da tecnologia no seu limite - processos de fabricação rápidos, controlados por computador, que permitem a repetição com variabilidade - exibe potenciais ainda por serem explorados, em especial no campo da habitação social, do design humanitário e na interseção entre alta e baixa tecnologia estratégias críticas contextuais para a arquitetura.

Assim, essa pesquisa em andamento se mostra atenta aos pressupostos do UNHCR no que tange práticas humanitárias em relação aos refugiados, às filosofias de alteridade atuais - que prezam por responsabilidade e justiça sociais e uma consciência global ecológica e comunitária -, e tenta alargar o limite do campo disciplinar da arquitetura e do design frente às questões dos refugiados.

Nos tempos atuais em que a dispersão forçada de pessoas pelo mundo se agrava é necessário que arquitetos, designers, professores e estudantes universitários se sensibilizem acerca do direito internacional dos refugiados. É premente pensar coletiva e colaborativamente com esses profissionais, instituições e organizações sobre abordagens estratégicas e eficazes de lidar com tais questões para que novas proposições sejam feitas e que mudanças de comportamento e atitudes de todos os atores envolvidos nos processos de inovação possam refletir de forma relevante nas políticas e ações humanitárias com vistas à reversão da situação dos refugiados.

\section{AGRADECIMENTOS}

Agradecemos ao FIP / PUC Minas - Fundo de Incentivo à Pesquisa pelo financiamento da pesquisa a partir da qual este artigo foi escrito; ao Instituto de Ciências Sociais e ao Departamento de Arquitetura e Urbanismo da PUC Minas; ao LEFAD - Laboratório de Experimentação em Fabricação Digital - da mesma instituição; ao Grupo de Pesquisa "Design e Fabricação Digital"; e aos professores Danny Zahreddine e Sergio de Lima Saraiva Junior pelo apoio.

\section{REFERÊNCIAS}

ACNUR. Termo de Referência - Cátedra Sérgio Vieira de Mello. $2016 . \quad$ Retrieved from: http://www.acnur.org/portugues/wpcontent/uploads/2018/01/ Termo_de_Refer\%C3\%AAncia_CSVM_2016.pdf

Aquilino, M. (ed) (2011). Beyond Shelter: Architecture and Human Dignity. New York: Metropolis Books.

Bauman, Z. (2017). Estranhos à Nossa Porta. Rio de Janeiro: Zahar.

Bell, B., Wakeford, K. (2008). Expanding Architecture: Design as Activism. New York: Metropolis Books.

Bloomer, K. (2000). The Nature of Ornament: Rhythm and Metamorphosis in Architecture. New York: W. W. Norton \& Company.
Boff, L. (2008). Ecologia, Mundialização, Espiritualidade. Rio de Janeiro: Record.

Capra, F, Luisi, P. (2014). A visão sistêmica da vida. São Paulo: Cultrix.

Carvalho, D. (2016). A condição ornamental: as origens e os fundamentos do ornamento arquitetural contemporâneo. Tese de Doutorado. Retrieved from: http://www.bibliotecadigital.ufmg.br/dspace/handle/1843/MM MD-AU8QR2.

Cary, J. (2017). Design for Good: A New Era of Architecture for Everyone. Washington: Island Press.

Charlesworth, E. (2015) Humanitarian Architecture: 15 stories of architects working after disaster. London: Routledge.

Chun, A., Brisson, I. (2015). Ground Rules in Humanitarian Design (AD Reader). New Jersey: Wiley.

Collier, P.; Betts, A. (2017). Refuge: Rethinking Refugee Policy in a Changing World. Oxford: Oxford University Press.

Fairs, M. (2016). UNHCR teams up with MIT's D-Lab to overhaul "very top-down" refugee design strategy. Retrieved from: https://www.dezeen.com/2016/07/05/united-nations-highcommission-for-refugees-mit-overhaul-refugee-designstrategy-corinne-gray-interview/

Gramazio, F, Kohler, M. (2014, June). Made by Robots: Challenging Architecture at a Larger Scale. [S.I.]: Academy Press, nd 229.

Heroux, J., Norris, T., Rube, K., Nadimi, V. (2017). The Case for Healthy Places. Project for Public Spaces, Inc. Retrieved from: http://cdn. plataformaurbana.cl/wpcontent/uploads/2017/02/healthy-places-pps.pdf.

Herscher, A. (2017). Displacements: Architecture and Refugee. New York: Sternberg Press.

Keep, J. (n/d). Make your own 3d delta printer for ceramic. Retrieved from: http://www.keep-art.co.uk/Self_build.html.

Kleinschmidt, K. In Radford, T. (2015, November). Refugee camps are the "cities of tomorrow", says humanitarian-aid expert. Retrieved from: https://www.dezeen.com/2015/11/23/refugee-camps-cities-oftomorrow-killian-kleinschmidt-interview-humanitarian-aidexpert/

Oslo manual (2005). Guidelines for collecting and interpreting innovation data. OECD/Eurostat, Retrieved from: https://www.oecd-ilibrary.org.

Radford, T. (2015, November). Refugee camps are the "cities of tomorrow", says humanitarian-aid expert. Retrieved from: https://www.dezeen.com/2015/11/23/refugee-camps-cities-oftomorrow-killian-kleinschmidt-interview-humanitarian-aidexpert/

Scott, F. (2016). Outlaw Territories: Environments of Insecurity/Architectures of Counterinsurgency. Cambridge, MA: MIT Press.

Schmal, P., Elser, O., Scheuermann, A. (2017). Making Heimat: Germany, Arrival Country: Atlas of Refugee Housing. Berlim: Hatje Cantz.

Spivak, G. (2012). An Aesthetic Education in the Era of Globalization. Cambridge, MA: Harvard University Press.

UNHCR (2014). Global Strategy for Settlement and Shelter: A Unhcr Strategy 2014-2018. Geneva: UNHCR Division of Programme Support and Management.

UNHCR (2016). Shelter Design Catalogue. Geneva: Division of Programme Support and Management.

UNHCR (2017a). UNHCR Emergency Handbook: Shelter Solutions. Retrieved from: https://emergency.unhcr.org/entry/111688/shelter-solutions

UNHCR (2017b). UNHCR Emergency Handbook: Shelter Solutions in Urban Areas. Retrieved from: 
https://emergency.unhcr.org/entry/112634/shelter-in-urbanareas

UNHCR (2017c). UNHCR Emergency Handbook: Manual Camp Planning Standards (Planned Settlements). Retrieved from: https://emergency.unhcr.org/entry/125657/camp-planningstandards-planned-settlements

UNHCR (2017d). UNHCR Emergency Handbook: Manual Site Planning for Camps. Retrieved from: https://emergency.unhcr.org/entry/111625/site-planning-forcamps
UNHCR (2017e). Innovation Service: A brief innovation glossary. 3p. Retrieved from: http://www.unhcr.org/innovation/wpcontent/uploads/2017/07/UNHCRInnovation-Glossary.pdf

VENTEVOGEL, P. (2017). Pieter Ventevogel on Mental Health of Refugees. UNHCR, the UN Refugee Agency. Retrieved from: https://www.youtube.com/watch?v=xcN63zG0zOM.

WASP project (n/d). http://www.wasproject.it. 